Trauma Berufskrankh 2010 · 12 [Suppl 1]:86-91 DOI 10.1007/s10039-009-1511-4

Online publiziert: 30 . Juli 2009

(c) Springer Medizin Verlag 2009
J.C. Katthagen · C. Voigt · H. Lill

Unfall- und Wiederherstellungschirurgie,

Diakoniekrankenhaus Friederikenstift gGmbH, Hannover

\title{
Arthroskopische Eingriffe am Schultergelenk
}

\section{Ambulante vs. stationäre Behandlung}

Arthroskopische Eingriffe zeichnen sich im Vergleich zu offenen Operationen durch ein geringeres Weichteiltrauma aus, hiermit verbunden sind eine niedrigere postoperative Schmerzintensität und ein erhöhter Patientenkomfort [16]. Die Schulterarthroskopie mit ihrem breiten Indikationsspektrum in Traumatologie und Sportmedizin ist in ihrer Bedeutung unumstritten. Doch welche Eingriffe eignen sich für ein ambulantes Vorgehen und welche müssen unter stationären $\mathrm{Be}$ dingungen durchgeführt werden?

\section{Ambulantes Operieren}

Ambulante Operationen zeigen in ihrer Zahl seit Jahren einen steigenden Trend. Mittlerweile finden etwa $37 \%$ aller Operationen unter ambulanten Bedingungen statt [4]. Kurze und komplikationsarme Eingriffe wie bestimmte Materialentfernungen und Arthroskopien, die Operation des Karpaltunnelsyndroms und die Entfernung von Haut- und Weichteiltumoren werden häufig ambulant durchgeführt.

Nicht jede Operation eignet sich jedoch für ein ambulantes Vorgehen. Grundvoraussetzung hierfür ist die Zulassung als
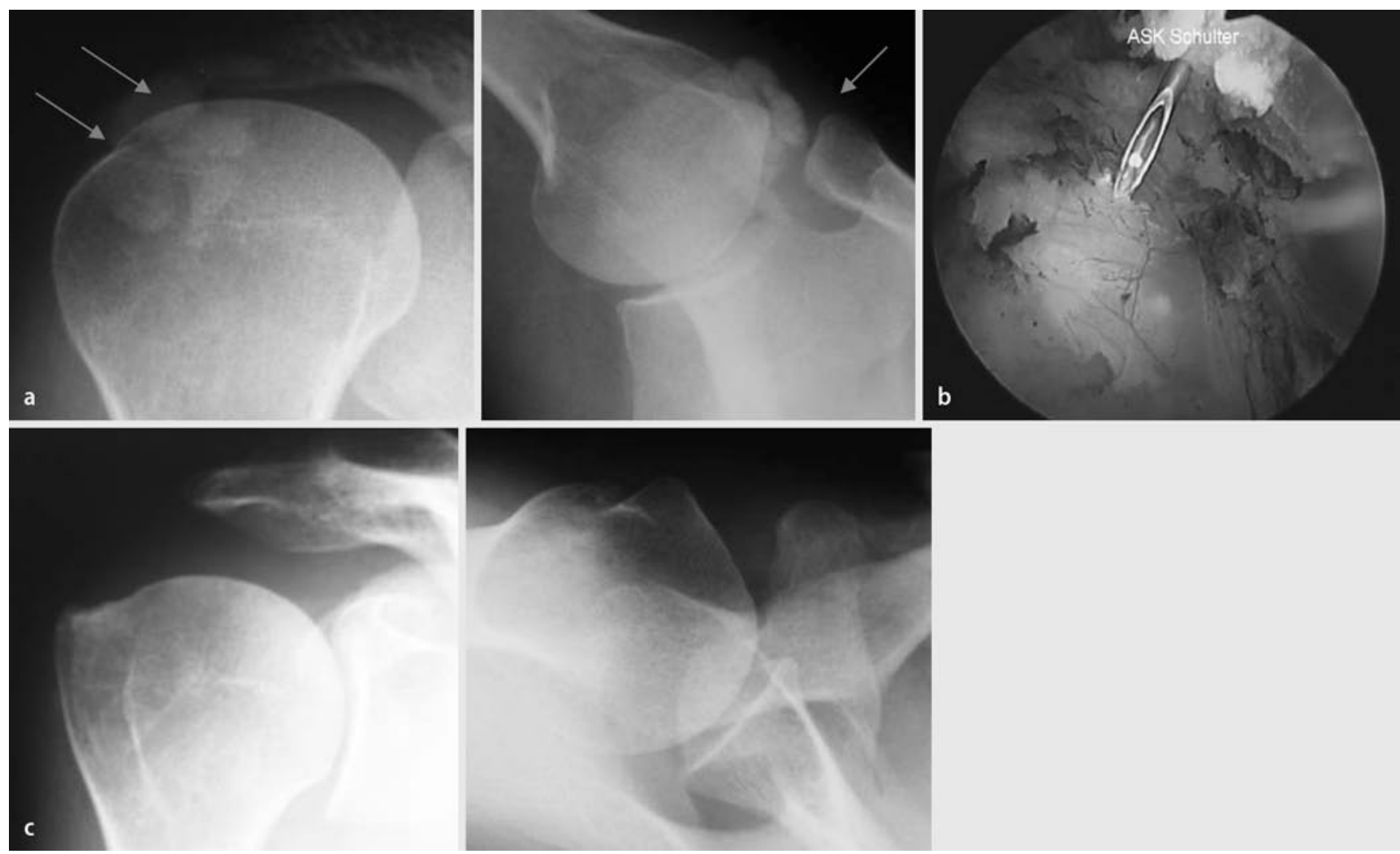

Abb. $1 \Delta$ Ausgeprägte Tendinitis calcarea der rechten Schulter, a präoperative Röntgenbilder a.-p. und axial, b rechte Schulter, Blick von dorsal, Lokalisation des Kalkdepots subakromial, c Röntgenbefund nach arthroskopischer Kalkentfernung a.-p. und axial 
ambulant durchführbare Operation gemäß \$115b des SGB V (Sozialgesetzbuch V). Bei der Entscheidungsfindung zwischen ambulantem und stationärem Operieren spielen die Möglichkeiten des intraund postoperativen Managements ebenso eine entscheidende Rolle wie die Gewährleistung einer adäquaten Schmerztherapie, einer kontinuierlichen Nachbehandlung sowie der häuslichen Versorgung des jeweiligen Patienten. Die Entfernung und Erreichbarkeit des nachbehandelnden Arztes und auch die schwierigere Terminkoordination unter ambulanten Bedingungen fließen in die Überlegungen mit ein. Die Möglichkeiten des ambulanten Operierens sind je nach bestehenden Vorerkrankungen und Risikofaktoren und bei unzureichender oder fehlender häuslicher Nachsorge eingeschränkt.

Die Indikation für eine ambulante Operation wird unter Abwägung der Größe des Eingriffs, dem Gesundheitszustand und sozialen Umfeld des Patienten sowie der Erfahrung des Operateurs gestellt [1]. Maßgeblich für den Erfolg und die Patientenzufriedenheit bei ambulanten Schulteroperationen sind die ausführliche und eingehende präoperative Aufklärung des Patienten über postoperative Abläufe und die Organisation der häuslichen Versorgung [5].

\section{Vor- und Nachteile ambulanter und stationärer Operationen}

\section{Ambulante Operationen}

Sie haben diverse Vorteile: Zum einen ist eine bessere Kontinuität der ärztlichen Versorgung gewährleistet, nach dem Prinzip „one client - one customer“. Der ambulant operierende Arzt begleitet den Patienten auf dem Weg von der Indikationsstellung und präoperativen Diagnostik zur Operation und nimmt auch die postoperative Nachbetreuung wahr [8]. Bei geringerem Spektrum ist ein standardisiertes, routiniertes und Zeit sparendes Vorgehen möglich. Eine anschließende Hospitalisierung ist nur selten notwendig, nosokomiale Infekte entfallen, und es zeigt sich ein geringeres Thromboserisiko im Vergleich zu stationären Operationen [7].

Trauma Berufskrankh 2010 - 12 [Suppl 1]:86-91 DOI 10.1007/s10039-009-1511-4

(c) Springer Medizin Verlag 2009

J.C. Katthagen $\cdot$ C. Voigt $\cdot$ H. Lill

Arthroskopische Eingriffe am Schultergelenk. Ambulante vs. stationäre Behandlung

\section{Zusammenfassung}

Die Zahl ambulanter Operationen steigt, dies gilt auch für die Schulterarthroskopie. Mit ihrem breiten Indikationsspektrum in Traumatologie und Sportmedizin ist sie unumstritten, eignet sich jedoch nicht in jedem Fall für ein ambulantes Vorgehen. Je nach Größe des Eingriffs, Gesundheitszustand und sozialem Umfeld des Patienten sowie Erfahrung des Operateurs muss individuell entschieden werden. Prinzipiell können nichtrekonstruktive Eingriffe wie die subakromiale Dekompression, die Akromioplastik, die Akromioklavikulargelenkresektion und die Kalkentfernung bei Tendinitis calcarea ambulant durch-

geführt werden. Rekonstruktive Eingriffe wie Stabilisierungen bei Schulterluxationen, Rekonstruktionen der Rotatorenmanschette oder Stabilisierungen des Akromioklavikulargelenkes sollten aufgrund der Größe der Eingriffe sowie des prä-, peri- und postoperativen Managements überwiegend unter stationären Bedingungen stattfinden.

\section{Schlüsselwörter}

Schulter · Arthroskopie - Ambulante Versorgung · Stationäre Versorgung .

Einzelfallentscheidung

\section{Arthroscopic shoulder surgery. Inpatient vs. outpatient treatment?}

\section{Abstract}

The number of outpatient operations increases; this is also true for shoulder arthroscopy. With its large range of indications shoulder arthroscopy is well established in trauma surgery and sports medicine. However, not all shoulder arthroscopies can be performed as an outpatient procedure. Each case needs to be decided individually on the basis of complexity of the procedure, the patient's general health and social environment, as well as the surgeon's experience. In general, non-reconstructive operations such as acromioplasty, subacromial decompression and excision of calcium for calcifying tendinitis can be carried out as outpatient procedures. Reconstructions of the rotator cuff or stabilisation after shoulder dislocation should be dealt with as inpatient procedures due to invasiveness of these operations as well as the pre-, peri- and postoperative management.

\section{Keywords}

Shoulder · Arthroscopy · Outpatient . Inpatient · Case-by-case decision-making 


\begin{tabular}{|c|c|c|}
\hline Eingriffsart & Indikation & Ambulant/stationär \\
\hline $\begin{array}{l}\text { Nichtrekonstruktive } \\
\text { Eingriffe }\end{array}$ & $\begin{array}{l}\text { Subakromiale Dekompression } \\
\text { Akromioplastik } \\
\text { AC-Gelenk-Resektion } \\
\text { Tendinitis calcarea }\end{array}$ & Ambulant \\
\hline $\begin{array}{l}\text { Rekonstruktive } \\
\text { Eingriffe }\end{array}$ & $\begin{array}{l}\text { Stabilisierung nach Luxation und chronischer } \\
\text { Instabilität } \\
\text { RM-Rekonstruktion } \\
\text { AC-Gelenk-Rekonstruktion } \\
\text { SLAP-Läsionen }\end{array}$ & Stationär \\
\hline $\begin{array}{l}\text { Revisionseingriffe/ } \\
\text { komplexe Eingriffe }\end{array}$ & $\begin{array}{l}\text { Arthrolyse } \\
\text { Arthroskopische Materialentfernung } \\
\text { Stabilisierung chronischer AC-Gelenk-Instabilitäten }\end{array}$ & Stationär \\
\hline
\end{tabular}

\section{Tab. 2 Klassifikation der SLAP-Läsionen. (Nach [17])}

Typ Charakteristika

I Degenerative Veränderungen des Labrumrandes ohne Beeinflussung der Verankerung von Bizeps und Labrum

II Abriss des Bizepssehnenankers vom Tuberculum supraglenoidale

III Korbhenkelriss des superioren Labrums, ohne dass der Bizepssehnenanker betroffen ist

IV Korbhenkelriss des superioren Labrums mit bis in den Bizepssehnenanker hinein reichendem Riss

V Abriss des Bizepssehnenankers vom Tuberculum supraglenoidale mit vorderer Kapsel-Labrum-Läsion (mit Übergang in Bankart-Läsion) ${ }^{\mathrm{a}}$

aTyp V: Teil der Erweiterung durch Maffet et al. [10]

Tab. 3 Klassifikation der Rotatorenmanschettenpartialrupturen. (Nach [6])

\begin{tabular}{ll} 
Grad & Durchmesser \\
I & $<1 / 4$ oder $<3 \mathrm{~mm}$ \\
II & $<1 / 2$ oder $3-6 \mathrm{~mm}$ \\
III & $>6 \mathrm{~mm}$ \\
\hline
\end{tabular}

Unerlässlich für ambulante Operationen sind gut geschultes medizinisches und pflegerisches Personal, um auftretende Probleme im weniger überwachten ambulanten Bereich früh zu erkennen und intervenieren zu können [1]. Atraumatisches und zügiges operatives Vorgehen auf Facharztniveau mit in der Folge geringerer Weichteilschwellung und niedrigerem Risiko der postoperativen Ergussbildung stellt eine Möglichkeit zur Verkleinerung des allgemeinen Komplikationsrisikos dar [1]. Weiterer entscheidender Faktor ist das möglichst optimale anästhesiologische Management mit allen Eventualitäten der regionalen und allgemeinen Anästhesiemethoden. Hierbei muss auch ambulant die postoperative Überwachung für einige Stunden gewährleistet sein.
Die fehlende Möglichkeit der lokalen Nervenblockade durch Schmerzkatheter im ambulanten Bereich stellt einen großen Nachteil mit in der Folge eingeschränkter postoperativer Schmerztherapie dar. Insbesondere bei größeren rekonstruktiven sowie Revisionseingriffen erscheint die fehlende Option der lokalen Nervenblockade, welche den Patientenkomfort deutlich verbessert [3], bereits als relative Kontraindikation für ein ambulantes Vorgehen.

\section{Stationäre Operationen}

Die Methodenvielfalt, sowohl operativ als auch anästhesiologisch, ist klarer Vorteil der stationären Operation. Unter besseren Überwachungsmöglichkeiten besteht bei Bedarf interdisziplinäre Hilfe, für den Patienten ist „immer jemand da“. Eine Vertretungsregelung für den Operateur wird unter stationären Bedingungen ermöglicht, bei Spezialfragen sind Spezialkenntnisse breiter gestreut [8]. Weiterhin sind Ressourcen für die Beherrschung systemischer und vitaler Komplikationen gegeben.

\section{Schulterarthroskopie - ambulant vs. stationär}

Zum breiten Indikationsspektrum der Schulterarthroskopie zählen u. a. das Impingementsyndrom, Rotatorenmanschettenrupturen, Stabilisierungen nach Luxationen sowie Verletzungen des Akromioklavikulargelenkes (AC-Gelenkes). Arthroskopien der Schulter lassen sich in nichtrekonstruktive, rekonstruktive und Revisionseingriffe unterteilen (• Tab. 1).

\section{Nichtrekonstruktive Eingriffe}

Hierunter fallen z. B. eine Kalkentfernung bei Tendinitis calcarea ( $\bullet$ Abb. 1), eine subakromiale Dekompression sowie eine Akromioplastik. Sie lassen sich aufgrund der Kürze der Operation, des geringen Operationstraumas und der geringeren Komplikationsrate gut unter ambulanten Bedingungen durchführen.

\section{Rekonstruktive Eingriffe}

Sie sollten stationär durchgeführt werden.

Stabilisierung nach traumatischer Schulterluxation. Sie ist gemäß $\$ 115 b$ SGB V eine ambulant durchführbare Operation. Die Ergebnisse und Komplikationsraten nach ambulanter Schulterstabilisierung sind mit denen nach stationärer Behandlung vergleichbar [11], wobei unter stationären Bedingungen jedoch eine bessere Analgesie und eine frühe physiotherapeutische Nachbehandlung möglich sind. Eine komplexe multidirektionale Stabilisierung sollte wegen der aufwändigen Rekonstruktion und Nachbehandlung in jedem Fall unter stationären Bedingungen erfolgen.

Stationär zu behandeln sind auch Schulterluxationen mit knöchernen Begleitverletzungen, da eine Osteosynthese am Glenoid, wie bei der Bankart-Fraktur, nicht zu den ambulant durchführbaren Operationen gemäß \$115b SGB V zählt.

Bei den ambulanten Operationen ist zu beachten, dass der Operateur in der Lage sein muss, „unerwartete intraoperative Befunde beherrschen zu können. Im konkreten Beispiel einer Schulterluxation gilt dies insbesondere für eine SLAPLäsion. Das Débridement einer SLAP-ILäsion nach Synder et al. ([17], - Tab. 2) 

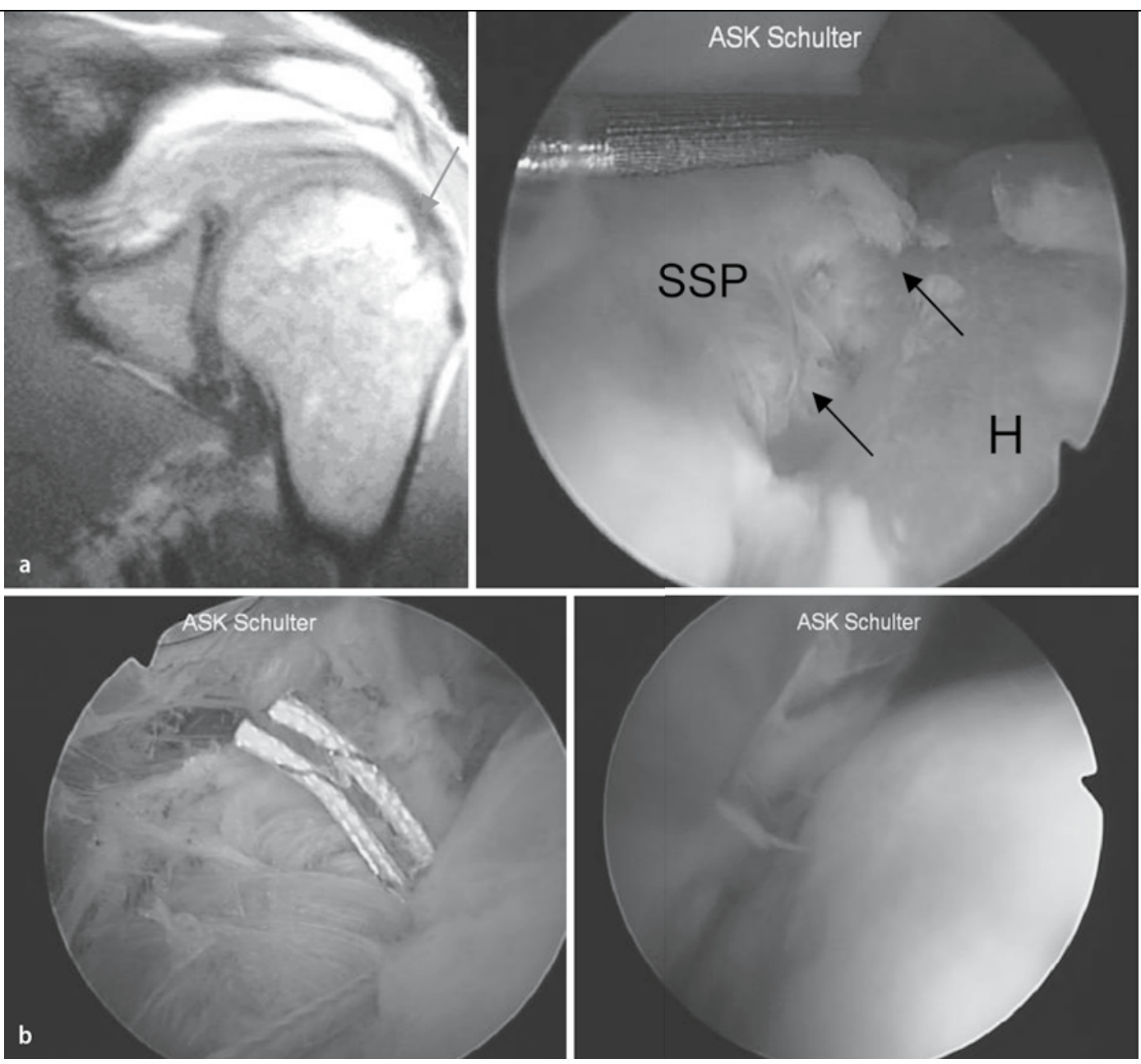

Abb. 2 a PASTA-Läsion Grad III nach Ellman [6], magnetresonanztomographisch und arthroskopisch (ASK), dorsale Pforte: Pfeile vom Humerus $(H)$ abgehobener Footprint (etwa $6 \mathrm{~mm}$ ) des Supraspinatusmuskels (SSP), b bursa- und artikularseitiges Ergebnis nach PASTA-Reparatur

lässt sich problemlos ambulant durchführen. Die Rekonstruktion höhergradiger SLAP-Läsionen dagegen sollte unter stationären Bedingungen stattfinden (- Tab. 1). Bei Techniken mit Refixation durch Fadenanker werden hierbei Ergebnisse mit guter Patientenzufriedenheit erzielt [15].

\section{Rotatorenmanschettenrekonstrukti-}

on. Sie ist nicht als ambulant durchführbare Operation im \$115b SGB V enthalten. Bei Partialrupturen Grad I und II nach Ellman ([6], - Tab. 3) lassen sich nach subakromialer Dekompression gute bis sehr gute Ergebnisse erzielen [9], eine Behandlung, welche unter ambulanten Bedingungen gut durchführbar ist. Bei Partialruptur Grad III nach Ellman ( Abb. 2a) ist eine PASTA-Reparatur (PASTA: „partial articular supraspinatus tendon avulsion“) notwendig ([19], - Abb. 2b). Sie zählt zu den rekonstruktiven Eingriffen und sollte demnach unter stationären Bedingungen erfolgen.
Komplettrupturen nach Bateman [2] sind nach neueren Erkenntnissen aufgrund der besseren Stabilität in DoubleRow-Technik zu rekonstruieren, hierbei zeigten sich signifikant geringere Rerupturraten $[12,13]$.

Die gesteigerten Implantatkosten bei rekonstruktiven Eingriffen sind bei der Frage der Kostendeckung im Rahmen einer ambulanten Operation nicht außer Acht zu lassen. Weiterhin sind die nicht unerheblichen Schmerzen des Patienten nach ambulanter Rotatorenmanschettenrekonstruktion nur schwer zu kontrollieren.

Bezüglich der ambulanten Rekonstruktion von Rotatorenmanschettenrupturen finden sich in der Literatur nur vereinzelte Daten aus den USA [5], welche eine ambulante Versorgung bei guter Patientenselektion für möglich und zumutbar halten. Die Übertragbarkeit der Daten auf unser ambulantes Gesundheitssystem, im Vergleich zu der engen Betreuung der Patienten durch den behandelnden Arzt in den USA, bleibt in Frage zu stellen [1]. Die
Hauptkomplikation unter 75 ambulant rekonstruierten Rotatorenmanschettenläsionen war starker postoperativer Schmerz [5], eine frühe intensive Schmerztherapie ist daher ratsam. Im Vergleich zu einem stationären Patientenkollektiv zeigten sich jedoch deutliche Unterschiede bezüglich des Alters und der Begleiterkrankungen der Patientengruppen [5].

Unbestritten ist, dass die Rekonstruktionen ausgeprägter Rotatorenmanschettenrupturen (z. B. 2-Sehnen-Läsionen von Supraspinatus- und Subskapularissehne) unter stationären Bedingungen erfolgen sollten.

AC-Gelenk-Rekonstruktion. Sie ist gemäß \$115b SGB V keine ambulant durchführbare Operation. Aufgrund der Bohrung durch Klavikula und Korakoid bestehen stärkere postoperative Schmerzen. Weiterhin treten in 15\% der RockwoodIII-Verletzungen traumatische glenohumerale Begleitverletzungen auf [14], die zeitgleich versorgt werden sollten. 


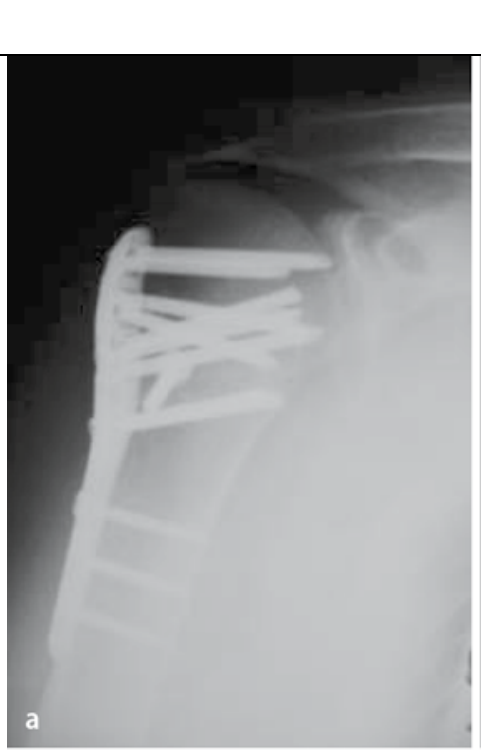

\section{Arthroskopische Eingriffe}
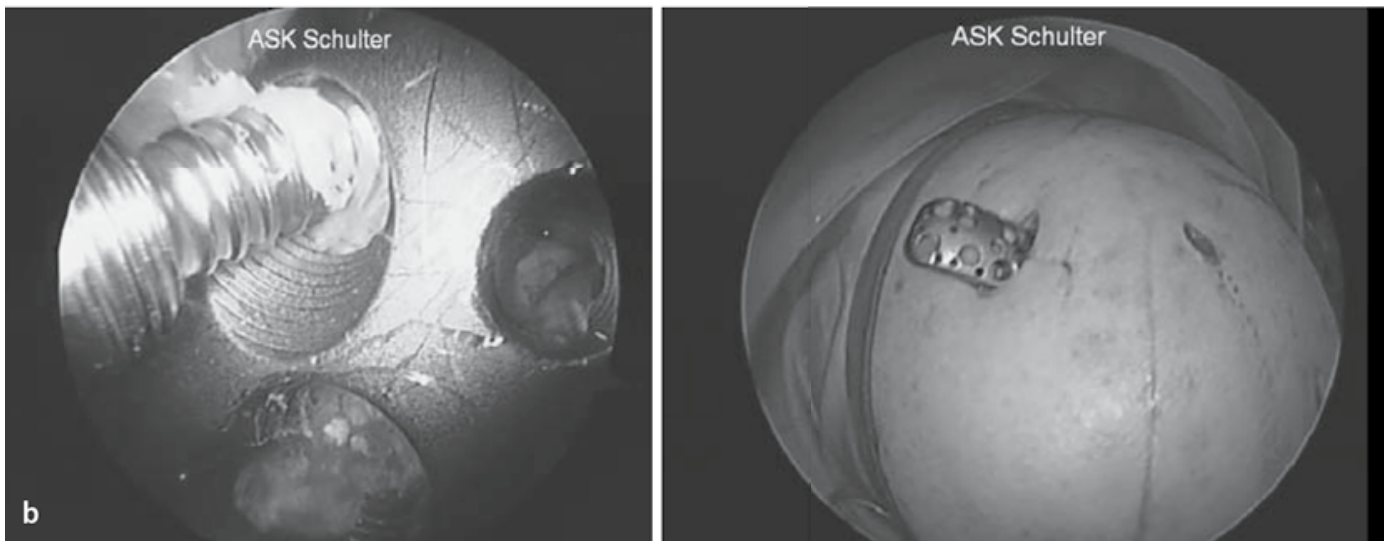

Abb. $3 \varangle$ a Vollständig konsolidierte proximale Humerusfraktur rechts mit perforierender Schraube, subakromial/subdeltoidal freigelegte Platte, Blick von dorsal, b arthroskopisches Entfernen der Schrauben und der Platte, ASK Arthroskopie

\section{Arthroskopische Revisionseingriffe} Sie sind an der Schulter mit einem Komplikationseingriff gleichzusetzen, da sie ein Patientenkollektiv betreffen, welches nach bereits erfolgter primärer Therapie weiterhin Beschwerdepersistenz aufweist. Dies gilt gleichermaßen für eine Arthrolyse bei Schultersteife, eine arthroskopische Materialentfernung am proximalen $\mathrm{Hu}$ merus sowie die AC-Gelenk-Rekonstruktion mittels Grafts bei chronischer ACGelenk-Instabilität.

Arthrolyse der Schulter. Sie ist gemäß \$115b SGB V ambulant durchführbar, erfordert jedoch für ein gutes Ergebnis eine intensive krankengymnastische Nachbehandlung, die direkt postoperativ beginnen sollte. Oft ist die Indikation für die Anlage eines Schmerzkatheters gegeben eine Kontraindikation für eine ambulante Behandlung.

Materialentfernung. Sie ist gemäß $\$ 115 b$ SGB V ebenfalls eine ambulant durchführbare Operation. Nach einer eigens entwickelten Technik ist die Entfernung einer Platte am proximalen Humerus auch arthroskopisch möglich ([18], - Abb. 3).

Die Materialentfernung wird bei persistierenden Bewegungseinschränkungen in Verbindung mit einer subakromialen und intraartikulären Arthrolyse oder bei perforierenden Schrauben durchgeführt und gehört somit zu den Revisionseingriffen (• Tab. 1). Als aufwändiger Eingriff mit notwendiger früher intensiver Nachbehandlung sollte die arthroskopische Plattenentfernung stationär durchgeführt werden.

\section{Fazit}

Allgemein übliche Empfehlungen für ambulante Schulterarthroskopien sind in der Literatur nur begrenzt vorhanden, da die jeweilige Entscheidung stark von der Klinik- oder Praxisstruktur, dem Operateur, den regionalen Gegebenheiten sowie dem Wunsch, Gesundheitszustand und individuellen sozialen Umfeld des jeweiligen Patienten abhängt.
Ambulant gut möglich sind die nicht rekonstruktiven Eingriffe. Rekonstruktive Eingriffe und Revisionsoperationen sollten aufgrund der Größe des Eingriffs und des prä-, peri- und postoperativen Managements mit adäquat angepasster Schmerztherapie sowie früher und intensiver krankengymnastischer Nachbehandlung unter stationären Bedingungen stattfinden.

\section{Korrespondenzadresse}

Prof. Dr. H. Lill

Unfall- und Wiederherstellungschirurgie, Diakoniekrankenhaus, Friederikenstift gGmbH, Humboldtstraße 5, 30169 Hannover Helmut.Lill@friederikenstift.de

Interessenkonflikt. Der korrespondierende Autor gibt an, dass kein Interessenkonflikt besteht.

\section{Literatur}

1. Agneskirchner JD, Lobenhoffer P (2004) Ambulante arthroskopische Chirurgie. Chirurg 75:233240 
2. Bateman JE (1963) The diagnosis and treatment of ruptures of the rotator cuff. Surg Clin North Am 43:1523-1530

3. Boezaart AP (2002) Continuous interscalene block for ambulatory shoulder surgery. Best Pract Res Clin Anaesthesiol 16:295-310

4. Brökelmann J, Reydelet J (2005) Zahl der Operationen in Deutschland 2003 - eine Annäherung. Ambulant Operieren 2:95-102

5. Cordasco FA, McGinley BJ, Charlton T (2000) Rotator cuff repair as an outpatient procedure. J Shoulder Elbow Surg 9(1):27-30

6. Ellman H (1990) Diagnosis and treatment of incomplete rotator cuff tears. Clin Orthop 254:64-74

7. Frank C, Krämer P, Reitzel T et al (2008) Etablierung einer ambulanten Operationseinheit. Ambul Chir 12(5):14-17

8. Hansis ML (2004) Qualitätssicherung beim ambulanten Operieren. Chirurg 75:120-125

9. Liem D, Alci S, Dedy N et al (2008) Clinical and structural results of partial supraspinatus tears treated by subacromial decompression without repair. Knee Surg Sports Traumatol Arthrosc 16(10):967-972

10. Maffet MW, Gartsmann GM, Moseley B (1995) Superior labrum-biceps tendon complex lesions of the shoulder. Am J Sports Med 23(1):93-98

11. Mayfield JB, Carter C, Wang C, Warner JJ (2001) Arthroscopic shoulder reconstruction: fast-track recovery and outpatient treatment. Clin Orthop 390:10-16

12. Ozbaydar M, Elhassan B, Esenyel C et al (2008) A comparison of single- versus double-row suture anchor techniques in a simulated repair of the rotator cuff: an experimental study in rabbits. J Bone Joint Surg Br 90(10):1386-1391

13. Park JY, Lhee SH, Choi JH et al (2008) Comparison of the clinical outcomes of single- and doublerow repairs in rotator cuff tears. Am J Sports Med 36(7):1310-1316

14. Pauly S, Gerhardt C, Haas NP, Scheibel M (2009) Prevalence of concomitant intraarticular lesions in patients treated operatively for high-grade acromioclavicular joint separations. Knee Surg Sports Traumatol Arthrosc 17(5):513-517

15. Samani JE, Marston SB, Buss DD (2001) Arthroscopic stabilization of type II SLAP lesions using an absorbable tack. Arthroscopy 17(1):19-24

16. Shibata T, Shiraoka K, Takubo N (1986) Comparison between arthroscopic and open synovektomie for the knee in rheumatoid arthritis. Arch Orthop Trauma Surg 105(5):257-262

17. Snyder SJ, Karzel RP, Del Pizzo W et al (1990) SLAP lesions of the shoulder. Arthroscopy 6(4):274-279

18. Voigt C, Geisler A, Lill H (2009) Arthroscopic locking plate removal after proximal humeral fractures. Arch Orthop Trauma Surg Apr 22. [Epub ahead of print]

19. Waibl B, Buess E (2005) Partial-thickness articular surface supraspinatus tears: a new transtendon suture technique. Arthroscopy 21(3):376-381 